\title{
On Positivity of Mass for Black Hole Space-Times
}

\author{
Pong Soo Jang \\ Theoretical Physics Institute, University of Alberta, Edmonton, Alberta, Canada T6G 2J1
}

\begin{abstract}
For any time-symmetric asymptotically flat initial slice with an apparent horizon, the associated four-momentum measured at spatial infinity is shown to be future-directed and time-like.
\end{abstract}

\section{Introduction}

Associated with any asymptotically flat space-time at spatial infinity there exists the notion of the total four-momentum, which is called Arnowitt-Deser-Misner (ADM) four-momentum [1-3]. If the asymptotically flat space-time is regular initially and matter fields are physically reasonable, one expects the ADM fourmomentum to be future-directed and time-like. This idea goes under the name of the positive mass conjecture (at spatial infinity) [4].

Recently, important progress has been made in this problem by Schoen and Yau [5], who showed that the ADM four-momentum is future-directed with respect to a regular maximal slice whose positive-definite metric satisfies a certain asymptotic condition. However, their proof fails to resolve the full conjecture because of two somewhat related reasons: First, as York [6] pointed out, their asymptotic condition on the positive-definite metric of the slice is too restrictive from the physical point of view. For the Schwarzschild solution, although their condition is satisfied on a $t=$ const surface, it is not satisfied on a boosted asymptotically flat slice. Second, note that even though the existence of a regular initial slice is assumed in the positive mass conjecture, singularities are allowed to develop in the future of the slice. This could cause additional difficulties in establishing the existence of a regular maximal slice. Even if one could overcome these difficulties, the proof of the conjecture requires one to show that the fourmomentum is time-like as well as future-directed. Only when the four-momentum has no spatial component with respect to a maximal slice, (e.g., when the slice is time-symmetric) is the positivity of energy, i.e., positivity of the time component of four-momentum, sufficient for the proof of the conjecture.

The full conjecture, thus, being still open, we would like to present another line of proof for a time-symmetric slice. In contrrast to Schoen and Yau's, our proof 
does not require such restrictive asymptotic conditions on the metric. Its other advantage is that energy can be shown to be positive even for time-symmetric slice which is singular inside an apparent horizon [7].

\section{Positive Mass Conjecture}

In this section we review the positive mass conjecture. For a space-time which represents an isolated system, one can define the total four-momentum of the system. In particular, the ADM four-momentum [1-3] is defined for space-times which are asymptotically flat at spatial infinity.

A space-time $\left(M, g_{a b}\right)$ is said to be asymptotically flat at spatial infinity if there exists a coordinate system $(t, x, y, z)$, with ranges, $-\infty<t<\infty$ and $r>r_{0}$, where $r=\sqrt{x^{2}+y^{2}+z^{2}}$ and $r_{0}$ is a large positive constant, so that with respect to this coordinate system the metric and the stress-energy tensor, $T^{a b}$, satisfy the following properties: The metric can be written as $g_{a b}=\eta_{a b}+k_{a b}$, where i) $\eta_{a b}=-\partial_{a} t \partial_{b} t+\partial_{a} x \partial_{b}+\partial_{a} y \partial_{b} y+\partial_{a} z \partial_{b} z$, ii) the components of $(r k),\left(r^{2} \partial k\right)$ and $\left(r^{3} \partial \partial k\right)$ have (finite) limits as $r$ goes to infinity keeping the ratios $(t / r, x / r, y / r, z / r)$ fixed such that $(t / r) \in(-1,1)$, iii) the $\operatorname{limits}, \lim (r k), \lim \left(r^{2} \partial k\right)$, and $\lim \left(r^{3} \partial \partial k\right)$, depend smoothly on the direction defined by the ratios, and iv) the components of $\left(r^{4} T\right)$ and $\left(r^{5} \partial T\right)$ have limits which depend smoothly on the direction.

Let $\tau$ be a smooth scalar field on an asymptotically flat space-time, with everywhere time-like gradient, and suppose the gradient has a (directiondependent) limit as $r$ goes to infinity. Let $\xi_{a}$ be a unit vector field on an asymptotically flat space-time so that the limit of $\xi_{a}$ exists and does not depend on the direction. Then, the ADM four-momentum, $P^{a}$, of the system observed with respect to a slice, $\tau=$ const, is defined as a linear mapping from the space of $\left(\lim \xi_{a}\right)$ to the reals in the following way [3],

$$
P^{a}\left(\lim \xi_{a}\right)=\lim _{r \rightarrow \infty}\left(-\frac{1}{8 \pi}\right) \int_{\substack{r=\text { const } \\ \tau=\text { const }}}\left(r C^{a b c d} \hat{r}_{b} \hat{r}_{d} \xi_{a} \hat{\tau}_{c}\right) d A,
$$

where $C^{a b c d}$ is the Weyl tensor of space-time, $\hat{\tau}_{a}$ is the unit vector normal to the slice $\tau=$ const, and $\hat{r}_{a}$ is the unit vector field normal to the surface $r=$ const in the slice, $\tau=$ const.

The idea of the positive mass conjecture is that this definition of total fourmomentum is reasonable in the sense that $P^{a}$ is future-directed and time-like if "the matter fields on $M$ is physically reasonable and $M$ is not too badly singular". Some conditions like these are clearly needed since the Schwarzschild solution with negative total mass is asymptotically flat (at spatial infinity). The conventional way to formulate the conjecture precisely is to impose suitable conditions on an asymptotically flat slice [4]. The slice, defined by $\tau=$ const, is said to be an asymptotically flat slice if the limit of $\partial_{a} \tau$ does not depend on the direction and $(\partial \partial \tau)$ has a direction dependent limit [3]. Then, the conjecture can be stated as follows :

Conjecture. If the stress-energy tensor satisfies the dominant energy condition [7] in an asymptotically flat space-time at spatial infinity, and if $M$ admits at least one 
asymptotically flat slice $\Sigma$ which is topologically $R^{3}$, then the ADM four-momentum $P^{a}$ is future-directed and time-like, and vanishes if and only if the space time is the Minkowski space.

Since $P^{a}$ is conserved, in the sense that the mapping from $\left(\lim \xi_{a}\right)$ to the reals does not depend on the choice of the slice $[2,3]$, it is sufficient to show the validity of the conjecture on the $R^{3}$-slice $\Sigma$ whose existence is assumed in the conjecture. In this way, the problem is reduced to a study of the initial data of Einstein's field equation.

On the asymptotically flat slice $\Sigma$, defined by $\tau=$ const, let $E$ denote the total energy defined by

$$
E=P^{a}\left(\lim \hat{\tau}_{a}\right)=\lim _{r \rightarrow \infty}\left(-\frac{1}{8 \pi}\right) \int_{\substack{r=\text { const } \\ \tau=\text { const }}}\left(r C^{a b c d} \hat{r}_{b} \hat{r}_{d} \hat{\tau}_{a} \hat{\tau}_{c}\right) d A .
$$

Since the 2-surfaces, defined by $r=$ const on $\Sigma$, approach a metric 2-sphere as $r$ goes to infinity, $E$ can be rewritten in the form (which we shall use in the following sections)

$$
E=\lim _{r \rightarrow \infty} \frac{1}{16 \pi} \int_{\substack{r=\text { const } \\ \tau=\text { const }}}\left[r\left(\tilde{R}-\tilde{p}^{2} / 2\right)+(r / 2)\left(\tilde{p}-\frac{2}{r \phi}\right)^{2}\right] d A,
$$

where $\tilde{R}$ and $\tilde{p}$ are, respectively, the intrinsic scalar curvature and the trace of the extrinsic curvature $\tilde{p}^{a b}$ of the integration surface considered as a submanifold of $\Sigma$, and $\phi=\left(D_{a} r D_{b} r q^{a b}\right)^{-1 / 2}$ where $q_{a b}$ is the metric of $\Sigma$ induced by $g_{a b}$. A similar expression to that of (3) was first given by Geroch [8]. Note that the integral of $r[\tilde{p}-2 /(r \phi)]^{2}$ does not contribute to $E$, since the integration surfaces approach a metric 2 -sphere so that $\lim r^{3}[\tilde{p}-2 /(r \phi)]^{2}=0$. The equivalence of this expression to (2) can be shown [9] using this freedom of adding terms which do not contribute to $E$ and the following identity

$$
\begin{aligned}
-C_{a b c d} \hat{\tau}^{b} \hat{\tau}^{d} \hat{r}^{a} \hat{r}^{c}= & \frac{1}{2}\left(\tilde{R}-\frac{1}{2} \tilde{p}^{2}\right)+\frac{1}{2}\left(\tilde{p}^{a b} \tilde{p}_{a b}-\frac{1}{2} \tilde{p}^{2}\right)-\frac{1}{2} p^{a b} p_{a b}+\frac{1}{2} p^{2}-8 \pi T^{a b} \hat{\tau}_{a} \hat{\tau}_{b} \\
& +p_{a}^{m} p_{b m} \hat{r}^{a} \hat{r}^{b}-p p_{a b} \hat{r}^{a} \hat{r}^{b}+\frac{8 \pi}{2}\left(T_{a b}-\frac{T}{3} g_{a b}\right)\left(q^{a b}+\hat{r}^{a} \hat{r}^{b}\right),
\end{aligned}
$$

where $p^{a b}$ is the extrinsic curvature of $\Sigma$ as a submanifold of space-time and $\tilde{p}^{a b}$ is the extrinsic curvature of the integration surface as a submanifold of $\Sigma$. All terms on the right hand side of the above equation, except the first one, do not contribute to $E$, (note $p^{a b}=O\left(1 / r^{2}\right)$ for an asymptotically flat slice [1-3]).

Suppose an asymptotically flat space-time admits a time-symmetric asymptotically flat slice $\Sigma^{\prime}$. Then, with respect to this slice, $P^{a}\left(\lim \xi_{a}\right)=0$ if $\left(\lim \xi_{a}\right)$ is orthogonal to $\left(\lim \hat{\tau}_{a}\right)$. Hence, the only thing we have to show in order to prove the positive mass conjecture for such a space-time is that $E$ is non-negative and vanishes if and only if the data on $\Sigma^{\prime}$ are those for Minkowski space. In the rest of the paper, we shall deal with the conjecture with the simplifying assumption that such a time-symmetric slice $\Sigma^{\prime}$ exists in the future or past of the $R^{3}$-slice $\Sigma$. We note that such a slice $\Sigma^{\prime}$ could be singular, since the initial data on $\Sigma$ could develop 
hypothesis [10] implies that any singularities on $\Sigma^{\prime}$ will be surrounded by apparent horizons. If this hypothesis is right, then the positive mass conjecture and the conservation of the ADM four-momentum predict that $E$ is positive for such a slice $\Sigma^{\prime}$ with an apparent horizon. Since whether this prediction is right seems to be interesting, we shall deal with this question explicitly in next sections. The modification of our argument for a time-symmetric (or maximal) slice with $R^{3}$ topology is not difficult.

\section{III. $E$ is Non-Negative for Time-Symmetric Data}

Let $\Sigma$ be a time-symmetric, (i.e. the extrinsic curvature $p^{a b}$ of $\Sigma$ vanishes), asymptotically flat slice with an apparent horizon $H$, that is, with a minimal 2-surface with spherical topology [11]. Even though $\Sigma$ could be singular inside $H$, outside $H$ we assume $\Sigma$ is regular and the region outside $H$ is diffeomorphic to $R^{3}$ minus a ball.

Now, the conditions of the positive mass conjecture imply the following properties of $\Sigma$ : The constraint equations of Einstein's equation and the dominant energy condition for the matter field imply that

$$
R=16 \pi T^{a b} \hat{\tau}_{a} \hat{\tau}_{b}+p^{a b} p_{a b}-p^{2} \geqq 0,
$$

where $R$ is the scalar curvature of the positive definite metric $q_{a b}$ of $\Sigma$. (Note that for a maximal slice we still have $R \geqq 0$.) Next, the asymptotic flatness of the spacetime and of the slice $\Sigma$ imply that the metric $q_{a b}$ of $\Sigma$ has the following asymptotic properties: There exists a coordinate system $(x, y, z)$ in which $q_{a b}$ can be written as $q_{a b}=\delta_{a b}+h_{a b}$, where i) $\delta_{a b}=\partial_{a} x \partial_{b} x+\partial_{a} y \partial_{b} y+\partial_{a} z \partial_{b} z$, ii) the components of $(r h)$, $\left(r^{2} \partial h\right)$, and $\left(r^{3} \partial \partial h\right)$ have limits as $r$ goes to infinity keeping the ratios $(x / r, y / r, z / r)$ fixed, iii) the limits depend smoothly on the direction defined by the ratios, and iv) $\left(r^{4} R\right)$ and the components of $\left(r^{5} \partial R\right)$ have limits which depend smoothly on the direction.

We now show that for such an asymptotically flat slice $\Sigma$ with an apparent horizon $H, E$ is positive.

Let $f$ be a positive solution of Laplace equation

$$
D^{a} D_{a} f=0
$$

with the boundary conditions, $f=f_{0}$ (where $f_{0}$ is a positive constant) on $H$ and $f=0$ at infinity, where $D_{a}$ is the covariant derivative with respect to $q_{a b}$. Such a solution exists [12]. Let the flux integral of $D_{a} f$ over a 2-surface, which encloses $H$, be $(-4 \pi e)$. We define a positive scalar field $r^{\prime}$ by

$$
r^{\prime}=e / f \text {. }
$$

Due to Coulomb-type behavior of $f$ in the asymptotic region, this $r^{\prime}$ can replace $r$ in the energy expression (3). From now on we shall drop the prime on $r^{\prime}$.

Let $W(c)$ denote the following integral

$$
\begin{aligned}
W(c) & =\frac{1}{16 \pi} \int\left[r\left(\tilde{R}-\frac{1}{2} \tilde{p}^{2}\right)+\frac{r}{2}\left(\tilde{p}-\frac{2}{r \phi}\right)^{2}\right] d A \\
& =\frac{1}{16 \pi} \int\left(r \tilde{R}+\frac{2}{r \phi^{2}}-\frac{2 \tilde{p}}{\phi}\right) d A
\end{aligned}
$$


over the 2-surface $(e / f)=r=c$, where $c$ is a constant. Let $\ell_{c}$ denote the set of points on which $r=c$. When $\ell_{c}$ does not contain critical points of $r$, i.e., the points at which $\left(D_{a} r\right)$ vanishes, $\ell_{c}$ is a sub-manifold of $\Sigma$ and $W(c)$ is well-defined. In particular, $W(c)$ is well-defined for large values of $c$, for $f$ (hence $r$ also) has no critical points in the asymptotic region. Further, we note that $\lim _{c \rightarrow \infty} W(c)=E$. The idea of our positive mass argument is, then, to establish the following theorem by examining the rate of change of $W(c)$ with respect to $c$.

Theorem. Let $\Sigma$ be a time-symmetric, asymptotically flat slice with an apparent horizon $H$. Let the region outside $H$ be diffeomorphic to $R^{3}$ minus a ball and $R \geqq 0$ there. Then $\lim _{c \rightarrow \infty} W(c)$ is positive.

In this section, we shall first establish this theorem for the special case where Laplace solution $f$ has no critical points. The generalization of the proof for $f$ with critical points will be carried out in the next section.

When $r$ does not have any critical point, the computation of $d W / d c$ is rather straightforward. We first observe that the Lie-derivative of $\tilde{p}$ along $\phi \hat{r}^{a}$ is given by

$$
\mathscr{L}_{\phi \hat{r}} \tilde{p}=-\frac{1}{2} \phi \tilde{p}^{a b} \tilde{p}_{a b}-\frac{1}{2} \phi \tilde{p}^{2}-\tilde{D}^{a} \tilde{D}_{a} \phi+\frac{1}{2} \phi \tilde{R}-\frac{1}{2} \phi R,
$$

where $\tilde{D}_{a}$ is the covariant derivative with respect to the intrinsic metric $\tilde{q}_{a b}$ of the integration surface. Since $(1 / r)$ satisfies Laplace's equation, we have

$$
\mathscr{L}_{\phi \hat{r}} \phi=\tilde{p} \phi^{2}-\frac{2 \phi}{r} .
$$

Now, using (9), (10) and Gauss-Bonnet theorem, we have

$$
\begin{aligned}
& \frac{d W}{d c}=\frac{1}{16 \pi} \int \tilde{R} d A+\frac{1}{16 \pi} \int \phi D_{a}\left[\left(\frac{2}{r \phi^{2}}-\frac{2 \tilde{p}}{\phi}\right) \hat{r}^{a}\right] d A \\
& \quad=\frac{1}{16 \pi} \int\left[R+\left(\tilde{p}^{a b} \tilde{p}_{a b}-\frac{1}{2} \tilde{p}^{2}\right)+2 \phi^{-2} \tilde{D}^{a} \phi \tilde{D}_{a} \phi+\frac{3}{2}\left(\tilde{p}-\frac{2}{r \phi}\right)^{2}+2 r^{2} \phi\left(\tilde{p}-\frac{2}{r \phi}\right)\right. \\
& \left.D^{2}\left(\frac{1}{r}\right)\right] d A .
\end{aligned}
$$

Since each term of the integrand is non-negative we have $d W / d c \geqq 0$. However, on the apparent horizon where $\tilde{p}$ vanishes, $W>0$. Therefore, we have $E=W(\infty)>0$.

In general, once we know that the integral of $\left(2 \tilde{R}-\tilde{p}^{2}\right)$ over a certain 2 -sphere in $\Sigma$ is non-negative, e.g., a very small sphere for an $R^{3}$-slice $\Sigma$ without a minimal 2-sphere, we can conclude that $E=W(\infty) \geqq 0$.

Note that in the computation of (11) we did not use the fact that all $\ell_{c}$ 's are 2 -spheres for $r$ without critical points. Hence, even for a regular $\ell_{c}$ which may be topologically different from a 2 -sphere we still have $d W / d c \geqq 0$.

\section{Case where Harmonic Function $f$ has Critical Points}

In this section, we generalize the argument of the previous section to the case when $f$ has critical points. This will be done in two steps. First, we shall deal with the 
simple case where the solution $f$ is strictly nondegenerate. Then, we shall deal with degenerate solutions. A function $f$ is said to be strictly nondegenerate if $D_{a} D_{b} f$ has an inverse at the critical points and if $\left.f\right|_{p} \neq\left. f\right|_{q}$ for every pair of critical points $p$ and $q[13]$.

\section{A. Case of Strictly Nondegenerate Solution $f$}

Assume the solution $f$ is strictly nondegenerate. As before, let $r$ denote e $/ f$. Since $f$ is positive the critical points of $f$ are the same as those of $r$. Then, as immediate consequences of $f$ being a solution of Laplace's equation, we have the following properties of the level surfaces $\ell_{c}$ [14]. If $\ell_{c}$ does not contain any critical point of $r$, $\ell_{c}$ is $a$ 2-sphere with a finite number of handles which encloses $H$. Also, each $\ell_{c}$ encloses those with lower value of $c$.

Near $H$, each $\ell_{c}$ is a 2 -sphere. Let $c_{1}$ be the smallest value at which $\ell_{c}$ has a critical point, say $q$. In terms of the normal coordinate, $x^{i}$, centered at $q$ the nondegenerate function $r$ can be written as [13]

$$
r=c_{1}+s_{i j} x^{i} x^{j}+O\left(\left|x^{i}\right|^{3}\right)
$$

where $s_{i j}$ is traceless symmetric matrix which has an inverse.

We now examine how $W(c)$ changes across $c_{1}$. That is to say, we compute the difference between $W\left(c_{1}+\right)=\lim _{c \rightarrow x_{1}+} W(c)$ and $W\left(c_{1}-\right)=\lim _{c \rightarrow c_{1}-} W(c)$. Recall that $W(c)$ is defined for $\ell_{c}$ with no critical points as

$$
W(c)=\frac{1}{16 \pi} \int_{\ell_{c}} r \tilde{R} d A+\frac{1}{16 \pi} \int_{\ell_{c}} \frac{2}{r}\left(q^{a b} D_{a} r D_{b} r\right) d A-\frac{1}{16 \pi} \int_{\ell_{c}} 2 \tilde{p}\left(q^{a b} D_{a} r D_{b} r\right)^{1 / 2} d A .
$$

The difference due to the first term of the right hand side can be obtained using Eq. (12) and the Gauss-Bonnet theorem. The Eq. (12) implies that $\ell_{c}$ changes from a 2 -sphere to a torus across $c_{1}$, hence we have

$$
\lim _{c \rightarrow c_{1}+} \int_{\ell_{c}} r \tilde{R} d A=\lim _{c \rightarrow c_{1}-} \int_{\ell_{c}} r \tilde{R} d A-8 \pi c_{1} .
$$

The change due to the second term is zero, since the integrand

$$
\left(2 r^{-1} q^{a b} D_{a} r D_{b} r\right)=O\left(\left|x^{i}\right|^{2}\right)
$$

near $q$. The integrand of the third term is $o\left(\left|x^{i}\right|^{-1}\right)$, hence the change due to this term is also zero. Therefore, we have

$$
W\left(c_{1}+\right)=W\left(c_{1}-\right)-\frac{c_{1}}{2} .
$$

In general, $W(c)$ decreases by $c_{i} / 2$ if the number of handles increases by one and $W(c)$ increases by $\left(c_{i} / 2\right)$ if the number of handles decreases by one, across critical value $c_{i}$.

Let $\tilde{W}(c)$ denote $\left[W(c)+c / 2\right.$ (number of handles of $\left.\left.\ell_{c}\right)\right]$. Then, the above observation can be stated as $\tilde{W}(c)$ does not change across critical value $c_{i}$. Furthermore, since we found in the previous section that $d W / d c \geqq 0$ for $\ell_{c}$ 's with no critical points, we have also $d \tilde{W} / d c \geqq 0$ for such $\ell_{c}$ 's. Hence, $\tilde{W}(c)$ is a never 
decreasing function of $c$. However, near the apparent horizon $H$ and in the asymptotic region, we have $W=\tilde{W}$. Therefore, from the monotonic property of $\tilde{W}(c)$ and the positivity of $W$ on $H$ we can conclude that $W(\infty)>0$.

\section{B. Case of Degenerate Solution $f$}

Finally, we deal with the case of a degenerate solution $f$. In a neighborhood of $H$ and in the asymptotic region, $f$ has no critical point. Let $\psi$ be a smooth function which is 1 in those regions and the interior of the support of $(\psi-1)$ includes all critical points of $f$. Let $\underline{f}$ denote a function outside $H$ defined by

$$
\underline{f}=\psi f+(1-\psi) v,
$$

where $v$ is a nondegenerate function. From Morse theory $[13,15]$ we have that for any small positive $\varepsilon$ there exists $v$ close enough to $f$ such that $\underline{f}$ is strictly nondegenerate and

$$
|f-\underline{f}|<\varepsilon, \quad\left|D^{a} D_{a} \underline{f}\right|<\varepsilon .
$$

We use such $\underline{f}$ to define $r$ as before, i.e., $r=e / \underline{f}$, where $(-4 \pi e)$ is the flux integral of $D_{a} f$. The idea of the proof is, then, to choose $\varepsilon$ small enough so that the additional negative contributions to $E$, due to the fact that $(1 / r)$ is no longer a Laplace solution, can be made arbitrarily small.

If the level surface $\ell_{c}$ of $r$ does not contain any critical point, then $\ell_{c}$ is a (or several) connected piece(s) each of which is a 2-sphere possibly with handles. Let $q$ be a critical point with critical value $c_{i}$. In terms of the normal coordinate, $x^{i}$, centered at $q$, the strictly nondegenerate $r$ can be written as

$$
r=c_{i}+s_{j k} x^{j} x^{k}+O\left(\left|x^{i}\right|^{3}\right)
$$

where $s$ is a symmetric matrix which has an inverse. Note $s$ does not need to be traceless. Hence, the point $q$ could be either a local minimum or maximum. Further, even when $s$ has an indefinite signature as before, the number of connected pieces in $\ell_{c}$ could change across $c_{i}$.

We first classify critical values as follows: Let " $I$ " denote the indefinite character of $s$ and " $D$ " denote the positive or negative definite character of $s$. Let $o$ affixed to $I$ represent the property that the number of connected pieces does not change as $c$ increases across $c_{i}$. Let $+(-)$ affixed to $I$ or $D$ represent the increase (decrease), by one, of the number of the connected pieces as $c$ increases. We, then, have five types of critical value, $I_{o}, I_{+}, I_{-}, D_{+}$, and $D_{-}$.

Each of these has the following properties:

Type $I_{o}$ : Critical values appeared in the strictly nondegenerate solution $f$ were all of this type. The only topological change in $\ell_{c}$ is the number of handles in $\ell_{c}$. If the signature of $s$ is $(+,-,-)$ then $W\left(c_{i}+\right)=W\left(c_{i}-\right)+c_{i} / 2$, and if $(+,+,-)$ then $W\left(c_{i}+\right)=W\left(c_{i}-\right)-c_{i} / 2$.

Type $I_{+}$: The signature of $s$ is $(+,-,-)$. For $c_{i}$ of this type, $W\left(c_{i}+\right)=W\left(c_{i}-\right)$ $+c_{i} / 2$.

Type $I_{-}:$The signature is $(+,+,-)$. For $c_{i}$ of this type, $W\left(c_{i}+\right)=W\left(c_{i}-\right)$ $-c_{i} / 2$.

Type $D_{+}$: The signature is $(+,+,+)$, that is, $q$ is a local minimum. We have $W\left(c_{i}+\right)=W\left(c_{i}-\right)+c_{i} / 2$. 
Type $D_{-}$: The signature is $(-,-,-)$, that is, $q$ is a local maximum. We have $W\left(c_{i}+\right)=W\left(c_{i}-\right)-c_{i} / 2$.

If $c$ is small, $\ell_{c}$ is near $H$ and is a 2 -sphere. As $c$ increases $\ell_{c}$ could become several connected pieces each of which is a 2 -sphere with handles. Suppose for $c_{n}<c<\left(c_{n}+\delta\right), \ell_{c}$ becomes a 2 -sphere again, for the first time. Such $c_{n}$ always exists since $\ell_{c}$ becomes a 2 -sphere in the asymptotic region. For simplicity, we assume that there is no more critical points for $c$ greater than $c_{n}$. (If there were more critical points, then repeating the following argument until $c$ crosses the largest critical value we can conclude the same.)

As for the strictly nondegenerate solution $f$, let $\tilde{W}(c)$ denote $[W(c)+c / 2$ (number of handles of $\ell_{c}$ )]. Note that the number of handles of $\ell_{c}$ changes only across critical values of type $I_{o}$. When it changes $W(c)$ changes in such a way that $\tilde{W}(c)$ does not change. Next, accounting the change of $W(c)$ across critical points of type $I_{+}, I_{-}, D_{+}$, and $D_{-}$and using the rate of change of $W(c)$ given by (11) for $\ell_{c}$ 's with no critical points we have

$$
W(\infty)=\tilde{W}(\infty)>\frac{1}{2} \sum_{i}\left( \pm c_{i}\right)+\frac{1}{8 \pi} \int d c \int_{\ell_{c}} d A\left[r^{2} \phi\left(\tilde{p}-\frac{2}{r \phi}\right) D^{2}\left(\frac{1}{r}\right)\right],
$$

where $c_{i}$ 's in the series are all of the type $I_{+}, I_{-}, D_{+}$or $D_{-}$.

The integral of the above inequality can be made as small as one wishes by choosing $\varepsilon$ of Eq. (17) small enough, thereby, making $\left|D^{2}(1 / r)\right|$ sufficiently small. We shall shortly show that the possible negative contribution from $\Sigma\left( \pm c_{i}\right)$ can be made as small as one wishes by choosing $\varepsilon$ small enough ${ }^{1}$. Once this has been done we can conclude $W(\infty)>0$.

Consider, now, the series of critical values of type $I_{+}, I_{-}, D_{+}$, and $D_{-}$. We shall pair these critical values such that the possible negative contribution from each pair is very small. First, for brevity, a connected regular 2-surface in $\ell_{c}$ which encloses $H$ will be said to be in the main sequence, $S$. Choosing $\varepsilon$ small enough we can assume that $c$ increases as we move outward from a surface in $S$, and that each $\ell_{c}$ without a critical point has one and only one connected 2-surface in $S$.

Consider the surface $\Gamma\left(\Gamma^{\prime}\right)$ of $\ell_{c_{k}+\delta}\left(\ell_{c_{k}-\delta}\right)$ in the main sequence $S$, where $c_{k}$ is a critical value. Let $d\left(d^{\prime}\right)$ denote the shortest distance from a point of $\Gamma\left(\Gamma^{\prime}\right)$ to $\Gamma^{\prime}(\Gamma)$. If both of $\underset{\Gamma}{\operatorname{Max}} d$ and $\underset{\Gamma^{\prime}}{\operatorname{Max}} d^{\prime}$ do not go to zero as $\delta$ goes to zero, we shall say $S$ is discontinuous at $c_{k}$. Note that at a critical point $c_{i}$ of type $I_{o}, S$ is not discontinuous.

Suppose $S$ is discontinuous at $c_{k}$. Then, the critical value $c_{k}$ should be of type $I_{+}$or $I_{-}$. Suppose $c_{k}$ is of type $I_{+}$. Then the level surface $\ell_{c_{k}+\varepsilon}$ should have a connected surface $\Gamma^{\prime}$ which does not belong to $S$ and whose interior has a local maximum point $q$ with critical value, say $c_{t}$. These two critical values appear in $\Sigma\left( \pm c_{i}\right)$ as $\left(+c_{k}\right)$ and $\left(-c_{t}\right)$. We evaluate $\left(c_{k}-c_{t}\right)$ as follows: Let $\varrho$ denote $(e / f)$. Then, since $f$ is a positive solution of Laplace equation, the maximum principle

1 It looks plausible that for any small $\varepsilon$ one can find a strictly nondegenerate $f$ such that Eq. (17) is satisfied and $f$ has critical points of type $I_{o}$ only. If this can be established we will not need this additional piece of argument 
[12] applies to $f$, hence to $\varrho$ also. Recall that $r=e / \underline{f}$. Using these facts and $|f-\underline{f}|<\varepsilon$, we obtain

$$
\begin{aligned}
\left(c_{k}+\varepsilon\right)-c_{t} & =\left[\left(c_{k}+\varepsilon\right)-\max _{\Gamma^{\prime}} \varrho\right]+\left[\max _{\Gamma^{\prime}} \varrho-\left.\varrho\right|_{q}\right]+\left[\left.\varrho\right|_{q}-c_{t}\right] \\
& \geqq-2 \varepsilon M+\left[\max _{\Gamma^{\prime}} \varrho-\left.\varrho\right|_{q}\right] \geqq-2 \varepsilon M,
\end{aligned}
$$

where $M$ is a positive constant determined by the maximum value of $(e / f)$ in the support of $(\psi-1)$. Hence, choosing $\varepsilon$ small enough, the possible negative contribution to $E$ from $\left(c_{k}-c_{t}\right)$ can be made as small as one wishes.

Suppose $c_{k}$ is of type $I_{-}$. Then, $\ell_{c_{k}-\varepsilon}$ has a connected surface which does not belong to $S$ and whose interior has a local minimum point $q$ with value, say $c_{t}$. By similar argument as for the case of $I_{+}$, we conclude the possible negative contribution from $\left(-c_{k}+c_{t}\right)$ is very small.

Next, let $S_{q}$ denote the sequence of regular connected 2-surfaces of $\ell_{c}$ each of which is not in $S$ and encloses the local maximum (or minimum) point $q$, whose critical value we just paired. This $S_{q}$ could be discontinuous at a critical value, say $c_{s}$. This $c_{s}$ is again of type $I_{+}$or $I_{-}$. If $c_{s}$ is of type $I_{+}$, there is a regular connected surface in $\ell_{c_{s}+\varepsilon}$ which does not belong to either $S$ or $S_{q}$ and whose interior has a local maximum point $q^{\prime}$ with value, say $c_{r}$. These critical values appear in the series $\Sigma\left( \pm c_{i}\right)$ as $\left(+c_{s}\right)$ and $\left(-c_{r}\right)$, and the possible negative contribution from $\left(c_{s}-c_{r}\right)$ is again very small. In case $c_{s}$ is of type $I_{-}$, there is an associated minimum point $q^{\prime}$ with value, say $c_{r}$, and the possible negative contribution from $\left(-c_{s}+c_{r}\right)$ is also very small.

We ask if any of $S, S_{q}$, and $S_{q^{\prime}}$ is discontinuous at a critical value other than those already paired. If so, we repeat the above described pairing with this new critical value. When all the sequences so far considered are not discontinuous at a new critical value, the sequences leave no open region outside $H$. Hence, all critical points of type $I_{+}, I_{-}, D_{+}$, and $D_{-}$are paired. The possible negative contribution from each pair can be made as small as one wishes by choosing $\varepsilon$ small enough, hence we conclude $E$ is positive.

Acknowledgments. I am grateful to Professors W. Israel, R. Geroch, D. Brill, and H. Künzle for valuable discussions and suggestions. This work has been supported by the Natural Sciences and Engineering Research Council of Canada.

\section{References}

1. Arnowitt, R., Deser, S., Misner, C.W.: Phys. Rev. 117, 1595-1602 (1960); 118, 1100-1104 (1960); 121, 1556-1566 (1961); 122, 997-1006 (1961); the article in : Gravitation, an introduction to current research. (ed. L. Witten) New York: Wiley 1962

2. Geroch, R.: J. Math. Phys. 13, 956-968 (1972); The article in: Asymptotic structure of space-time. (eds. F.P. Esposito, L. Witten) New York : Plenum 1976

3. Ashtekar, A., Hansen, R.D. : J. Math. Phys. 19, 1542-1566 (1978). Also see Ashtekar, A. : The article in : Einstein centennial volumes. (ed. A. Held) New York: Plenum 1979

4. See, for example, the review article by Brill, D. and Jang, P.S. in : Einstein centennial volumes. (ed. A. Held) New York: Plenum 1979, and references therein

5. Schoen, R., Yau, S.T.: Commun. Math. Phys. 65, 45-76 (1979)

6. York, J.: Has the positive energy conjecture been proved? Preprint 
7. For definition, see, for example, Hawking, S.W., Ellis, G.F.R.: The large scale structure of spacetime. Cambridge: Cambridge University Press 1973

8. Geroch, R.: Ann. N.Y. Acad. Sci. 224, 108-117 (1973)

9. Goldberg, J.: The article in: Einstein centennial volumes. (ed. A. Held) New York: Plenum 1979

10. Penrose, R.: Ann. N.Y. Acad. Sci. 224, 125-134 (1973). Also see Gibbons, G.: Commun. Math. Phys. 27, 87-102 (1972)

11. See Gibbons in [10]

12. Serrin, J., Weinberger, H.F.: Am. J. Math. 88, 258-272 (1966). Also see Meyers, N., Serrin, J. : J. Math. Mech. 9, 513-538 (1960)

13. Bott, R.H. : Lectures on Morse theory. University Bonn 1960

Milnor, J.W.: Morse theory. Princeton: Princeton University 1963

14. Müller zum Hagen, H. : Proc. Camb. Phil. Soc. 68, 187-197 (1970)

15. Albert, J.H. : Proc. Symp. Pure Math. 23, 71-78 (1973)

Communicated by R. Geroch

Received May 18, 1979 